\title{
The Chern-Simons Invariant in the Berry Phase of a Two by Two Hamiltonian
}

\author{
Wu-Yi Hsiang \\ Department of Mathematics, Hong-Kong University of Science and \\ Technology, Clear Water Bay, Kowloon, Hong Kong. \\ and \\ Dung-Hai Lee \\ Department of Physics, University of California, Berkeley, CA 94720.
}

\begin{abstract}
The positive (negaive)-energy eigen vectors of the two by two Hamiltonian $H=\mathbf{r} \cdot \vec{\sigma}$ where $\vec{\sigma}$ are the Pauli matrices and $\mathbf{r}$ is a 3-vector, form a $\mathrm{U}(1)$ fiber bundle when $\mathbf{r}$ sweeps over a manifold $\mathcal{M}$ in the three dimensional parameter space of $\mathbf{r}$. For appropriately chosen base space $\mathcal{M}$ the resulting fiber bundle can have non-trivial topology. For example when $\mathcal{M}=S^{2} \equiv\{\mathbf{r} ;|\mathbf{r}|=1\}$ the corresponding bundle has a non-zero Chern number, which is the indicator that it is topologically non-trivial. In this paper we construct a two by two Hamiltonian whose eigen bundle shows a more subtle topological non-triviality over $\mathcal{M}=R^{3} \bigcup\{\infty\}$, the stereographic projection of $S^{3}$. This non-triviality is characterized by a non-zero Chern-Simons invariant.
\end{abstract}

Since it's discovery in 1984 [1], Berry's phase has played an important role in quantum mechanics. For a simple example where Berry's phase occurs, consider the following two-level Hamiltonian

$$
H(\mathbf{r})=x \sigma_{x}+y \sigma_{y}+z \sigma_{z} \equiv \mathbf{r} \cdot \vec{\sigma}
$$

where $\sigma_{x, y, z}$ are the three components of the Pauli matrices and $x, y, z$ are three real parameters. For a fixed $\mathbf{r}=(x, y, z)$ the Hamiltonian given in Eq. (11) has two eigenvalues $E_{ \pm}= \pm|\mathbf{r}|$. Let us denote the corresponding normalized two-component eigen vectors by $\mid \psi_{ \pm}(\mathbf{r})>$.

For the rest of the paper let us focus on, say, the positive-energy eigen vector $\left|\psi_{+}(\mathbf{r})\right\rangle$. Since the transformation $\left|\psi_{+}(\mathbf{r})>\rightarrow e^{i \theta}\right| \psi_{+}(\mathbf{r})>$ preserves the normalization and the eigen nature of $\left|\psi_{+}(\mathbf{r})\right\rangle$, there is a continuum of two-component vectors, each labeled by $\theta$, satisfying $H\left|\psi_{+, \theta}(\mathbf{r})>=\right| \mathbf{r} \| \psi_{+, \theta}(\mathbf{r})>$. For a given $\mathbf{r}$ the internal space formed by all $\mid \psi_{+, \theta}(\mathbf{r})>$ has the $\mathrm{U}(1)$ group structure. When $\mathbf{r}$ varies over a manifold $\mathcal{M}$ (henceforth referred as the base space) the internal space sweeps out a "fiber bundle". Since the internal space has $\mathrm{U}(1)$ symmetry this fiber bundle is called a $\mathrm{U}(1)$ bundle. In the following we shall refer to such fiber bundle as the eigen bundle of Eq. (1).

A connection can be defined on the eigen bundle by first choosing a reference eigen vector $\mid \psi_{+}(\mathbf{r})>$ at each $\mathbf{r}$ then defining the vector field

$$
\mathbf{A}_{b}(\mathbf{r})=\frac{1}{i}<\psi_{+}(\mathbf{r}) \mid \nabla \psi_{+}(\mathbf{r})>.
$$

We note that in order for $\mathbf{A}_{b}$ to be well defined, the reference vector must vary continuously with $\mathbf{r}$. The connection $\mathbf{A}_{b}$ defined above is not unique. Indeed, by performing the transformation $\left|\psi_{+}(\mathbf{r})>\rightarrow e^{i \theta(\mathbf{r})}\right| \psi_{+}(\mathbf{r})>$ we induce a "gauge transformation" on $\mathbf{A}_{b}$ :

$$
\mathbf{A}_{b} \rightarrow \mathbf{A}_{b}+\nabla \theta .
$$

The Berry's phase caused by an adiabatic evolution of $\mathbf{r}$ around a closed loop $\mathcal{C}$ is given by

$$
\gamma=\oint_{\mathcal{C}} d \mathbf{r} \cdot \mathbf{A}_{b}(\mathbf{r})
$$

Obviously the Berry's phase is gauge invariant.

Let us first consider two-dimensional base spaces $\mathcal{M}$ that are closed surfaces. It turns out that if $\mathcal{M}$ encloses the origin (for example $\mathcal{M}=S^{2}=\{\mathbf{r} ;|\mathbf{r}|=1\}$ ), it is impossible to choose a gauge in which $\mathbf{A}_{b}(\mathbf{r})$ is nonsingular everywhere. In order to obtain non-singular $\mathbf{A}_{b}$ it is necessary to divide $\mathcal{M}$ into a number of (overlapping) patches so that 1) $\mathbf{A}_{b}$ is non-singular in each patch, and 2) in the overlapping region of two patches the different $\mathbf{A}_{b}$ 's only differ by a gauge transformation. Historically a problem like this was encountered when Dirac tried to write down the vector potential in the surrounding of a magnetic monopole. [2] It turns out that under the framework of quantum mechanics the condition 2) stated above requires the strength of the monopole to be quantized. [2, 3]

The fact that it is impossible to define an everywherenonsingular connection is the symptom of non-trivial topology. In his seminal work Chern discovered a set of invariants to characterize such non-triviality. [4] For the simple case we are considering the invariants reduce to a single number, the Chern number:

$$
\mathcal{C}=\frac{1}{4 \pi} \int_{\mathcal{M}} d \mathbf{a} \cdot \mathbf{B}_{b}
$$

Here $\mathbf{B}_{b}=\nabla \times \mathbf{A}_{b}$ is the curvature associated with $\mathbf{A}_{b}$. ( Note that in order to have a well defined $\mathbf{B}_{b}$ we need a locally differentiable $\mathbf{A}_{b}$.) For the eigen bundle of Eq. (1) Berry has shown that [1] 


$$
\mathbf{B}_{b}(\mathbf{r})=\frac{1}{2} \frac{\hat{r}}{|\mathbf{r}|^{2}}
$$

The right hand side of Eq. (6) is the same as the magnetic field produced by a monopole sitting at the origin. Thus if $\mathcal{M}$ encloses the origin Eq. (阿) yields $\mathcal{C}=1 / 2$ indicating the corresponding eigen bundle is non-trivial. On the other hand if $\mathcal{M}$ does not enclose the origin, then $\mathcal{C}=0$ and the corresponding eigen bundle is trivial. The Chern number (Eq. (5)) has a simple physical interpretation - it is the total flux produced by the bundle curvature. In a proof similar to that given in Ref. [3], Chern showed that $\mathcal{C}$ should be quantized to values $n / 2$ where $n=$ integer. 叫

Since the Chern number can be larger than $1 / 2$, it is interesting to know what kind of Hamiltonian will exhibit $\mathcal{C}=n / 2(n>1)$ eigen bundles.

One answer is given by the following $(n+1) \times(n+1)$ matrix

$$
H(\mathbf{r})=\mathbf{r} \cdot \mathbf{S},
$$

where $\mathbf{S}=\left(S_{x}, S_{y}, S_{z}\right)$ are the matrices representing the three generators of $\mathrm{SU}(2)$ in the higher spin $(S=n / 2)$ representation. For example for $n=2$ we have

$$
\begin{aligned}
S_{x} & =\frac{1}{\sqrt{2}}\left(\begin{array}{ccc}
0 & 1 & 0 \\
1 & 0 & 1 \\
0 & 1 & 0
\end{array}\right), S_{y}=\frac{1}{\sqrt{2}}\left(\begin{array}{ccc}
0 & -i & 0 \\
i & 0 & -i \\
0 & i & 0
\end{array}\right) \\
S_{z} & =\left(\begin{array}{ccc}
1 & 0 & 0 \\
0 & 0 & 0 \\
0 & 0 & -1
\end{array}\right) .
\end{aligned}
$$

There is another modification of Eq. (11) which also leads to $\mathcal{C}=n / 2$ eigen bundle. Interestingly this time we do not need to enlarge the dimension of the Hamiltonian matrix. Consider the following $2 \times 2$ matrix

$$
H(\mathbf{r})=\hat{h}(\mathbf{r}) \cdot \vec{\sigma},
$$

where $\hat{h}(\mathbf{r})$ is a suitably chosen unit vector field that defines a mapping from $\mathcal{M}$ (a closed two-dimensional surface) to $S^{2}$. It is known that such mappings can be classified into homotopy classes each labeled by an integer

$$
\mathcal{P}=\int_{\Gamma} d \mathbf{a} \cdot \mathbf{J} .
$$

Here $\mathbf{J}$, the dual of the Pontryagin form, is given by

$$
J^{\mu}=\frac{1}{8 \pi} \epsilon^{\mu \nu \lambda} \hat{h} \cdot\left(\partial_{\nu} \hat{h} \times \partial_{\lambda} \hat{h}\right) .
$$

We will show later that by choosing a $\hat{h}(\mathbf{r})$ with $\mathcal{P}=n$ in Eq. (9) the corresponding eigen bundle has Chern number $\mathcal{C}=n / 2$.

The Chern number records the highest level of topological non-triviality. When the Chern number vanishes the eigen bundle can still be non-trivial at a more subtle level. Let us consider a three-dimensional base space in which $\mathcal{C}=0$ for all closed surfaces, implying the absence of "B $\mathbf{B}_{b}$-monopole". In that case the "flux lines" of $\mathbf{B}_{b}$ form closed loops. There is a topological interesting situation in which these flux lines link with one another. It is clear that this class of eigen bundles are topologically distinct from those whose curvature flux loops do not link.

In 1974 Chern and Simons discovered an invariant, the Chern-Simons invariant, that quantifies this more subtle topological non-triviality. [5] For a three-dimensional manifold $\mathcal{M}$ the Chern-Simons invariant is given by

$$
\mathcal{C S}=\frac{1}{4 \pi} \int_{\mathcal{M}} d^{3} r \mathbf{A}_{b} \cdot \nabla \times \mathbf{A}_{b} .
$$

In order for $\mathcal{C S}$ to be gauge invariant we require $\mathcal{M}$ to be free of boundary. The topological information recorded by $\mathcal{C S}$ is precisely the linking between the $\mathbf{B}_{b}$ flux lines. The fact that linking is only defined in three dimensions explains why the Chern-Simons invariant requires a three dimensional base space.

After realizing that there is another level of topological non-triviality it is natural to ask whether one can modify Eq. (1) so that the eigen bundle exhibits non-zero $\mathcal{C S}$. We shall prove that the Hamiltonian given by Eq. (9) also works so long as $\hat{h}(\mathbf{r})$ is chosen appropriately.

Now let us restrict ourselves to the case where the base space $\mathcal{M}$ is $R^{3} \cup\{\infty\}$, the stereographic projection of $S^{3} \equiv\left\{(x, y, z, w) ; x^{2}+y^{2}+z^{2}+w^{2}=1\right\}$. In that case $\hat{h}(\mathbf{r})$ is a mapping from $R^{3} \bigcup\{\infty\}$ to $S^{2}$. Due to the work of Hopf it is known that such mapping can also be classified into homotopy classes. There also exists an integer, the Hopf invariant, that characterizes each class. The meaning of a non-trivial Hopf map is revealed by the dual of its Pontryagin form (Eq. (11)). For both the trivial and non-trivial Hopf maps $\nabla \cdot \vec{J}=0$ everywhere hence the $\mathbf{J}$ flux lines form closed loops. For a non-trivial map the closed flux loops link with one another while for a trivial map they don't. Now we explain what is the Hopf invariant. Since $\nabla \cdot \mathbf{J}=0$, there exists a vector field $\mathbf{A}_{h}$ so that

$$
\mathbf{J}=\frac{1}{2 \pi} \nabla \times \mathbf{A}_{h}
$$

The Hopf invariant is simply the Chern-Simons invariant for $\mathbf{A}_{h}$, [6] i.e.,

$$
\mathcal{H}=\frac{1}{4 \pi} \int_{\mathcal{M}} d^{3} r \mathbf{A}_{h} \cdot \nabla \times \mathbf{A}_{h} .
$$

In Fig.1 we plot $\mathbf{J}$ in the central region of the following $\mathcal{H}=1$ map [7]:

$$
\begin{aligned}
& \mathbf{r}=r(\sin \theta \cos \phi, \sin \theta \sin \phi, \cos \theta) \\
& \hat{h}=(\sin \beta \cos \alpha, \sin \beta \sin \alpha, \cos \beta)
\end{aligned}
$$




$$
\begin{aligned}
& \alpha(\mathbf{r})=\Theta\left(r^{2}-1+i 2 r \cos \theta\right)-\phi \\
& \beta(\mathbf{r})=2 \Theta\left(\sqrt{\left(1-r^{2}\right)^{2}+4 r^{2} \cos ^{2} \theta}+i 2 r|\sin \theta|\right) .
\end{aligned}
$$

In the above $\Theta(u+i v)$ is the angle of the $\mathrm{U}(1)$ phase factor $(u+i v) /|u+i v|$. The green and blue arrows mark the $\mathrm{x}, \mathrm{y}$ and $\mathrm{z}$ components of $\mathbf{J}(\mathbf{r})$ respectively. The purpose of this plot is to illustrate that the green arrows loop around the blue ones hence manifests the linking in of the $\mathbf{J}$ lines.

In the rest of the paper we prove the following.

1. For base space $\mathcal{M}=S^{2}$ the eigen bundle of $E q$. (9) has $\mathcal{C}=n / 2$ if $\hat{h}(\mathbf{r})$ has $\mathcal{P}=n$.

2. For base space $\mathcal{M}=R^{3} \bigcup\{\infty\}$ the eigen bundle of

Eq. (9) has $\mathcal{C S}=n$ if $\hat{h}(\mathbf{r})$ has $\mathcal{H}=n$.

The proof rests on the identity that

$$
\mathbf{B}_{b}=2 \pi \mathbf{J}
$$

which we now show. The Berry curvature is given by

$$
\mathbf{B}_{b}=\frac{1}{i} \nabla \times<\psi_{+} \mid \nabla \psi_{+}>
$$

Since $<\psi_{+} \mid \psi_{+}>=1$, it follows that $<\nabla \psi_{+} \mid \psi_{+}>$ $+<\psi_{+} \mid \nabla \psi_{+}>=0$. Furthermore since $<\nabla \psi_{+} \mid \psi_{+}>=<$ $\psi_{+} \mid \nabla \psi_{+}>^{*}$ we conclude that $<\psi_{+} \mid \nabla \psi_{+}>$is pure imaginary. Thus

$$
\begin{aligned}
& \mathbf{A}_{b}=\operatorname{Im}\left[<\psi_{+} \mid \nabla \psi_{+}>\right] \\
& \mathbf{B}_{b}=\operatorname{Im}\left[\nabla \times<\psi_{+} \mid \nabla \psi_{+}>\right] .
\end{aligned}
$$

In component form the second of Eq. (20) read

$$
\begin{aligned}
B^{\mu} & =\epsilon^{\mu \nu \lambda} \operatorname{Im}\left[<\partial_{\nu} \psi_{+} \mid \partial_{\lambda} \psi_{+}>\right] \\
& =\frac{\epsilon^{\mu \nu \lambda}}{2} \operatorname{Im}\left[<\partial_{\nu} \psi_{+}\left|\partial_{\lambda} \psi_{+}>-<\partial_{\lambda} \psi_{+}\right| \partial_{\nu} \psi_{+}>\right]
\end{aligned}
$$

To compute $<\partial_{\nu} \psi_{+}\left|\partial_{\lambda} \psi_{+}>-<\partial_{\lambda} \psi_{+}\right| \partial_{\nu} \psi_{+}>$we insert a complete set of states $\left(I=\sum_{n= \pm}\left|\psi_{n}><\psi_{n}\right|\right)$, and that gives

$$
\begin{aligned}
& <\partial_{\nu} \psi_{+}\left|\partial_{\lambda} \psi_{+}>-<\partial_{\lambda} \psi_{+}\right| \partial_{\nu} \psi_{+}> \\
& =\sum_{n= \pm}<\partial_{\nu} \psi_{+}\left|\psi_{n}><\psi_{n}\right| \partial_{\lambda} \psi_{+}>-[\nu \leftrightarrow \lambda] \\
& =<\partial_{\nu} \psi_{+}\left|\psi_{-}><\psi_{-}\right| \partial_{\lambda} \psi_{+}>-[\nu \leftrightarrow \lambda] .
\end{aligned}
$$

In reaching the last line we have used the fact that $<\partial_{\nu} \psi_{+}\left|\psi_{+}><\psi_{+}\right| \partial_{\lambda} \psi_{+}>-[\nu \leftrightarrow \lambda]=0$.

To compute $\left\langle\psi_{-}\right| \partial_{j} \psi_{+}>$in Eq. (22) we express the eigen vector of $H\left(\mathbf{r}^{\prime}\right)=H(\mathbf{r}+\delta \mathbf{r})=H(\mathbf{r})+\delta \mathbf{r} \cdot \nabla H$ in terms of those of $H(\mathbf{r})$ via first order perturbation theory. To the first order in $|\delta \mathbf{r}|$ we obtain

$$
\begin{aligned}
\mid \psi_{+}\left(\mathbf{r}^{\prime}\right)> & =\left[\left|\psi_{+}>+\frac{\left\langle\psi_{-}|\delta \mathbf{r} \cdot \nabla H| \psi_{+}>\right.}{E_{+}-E_{-}}\right| \psi_{-}>\right] \\
& =\left[\left|\psi_{+}>+\frac{<\psi_{-}\left|\delta r_{\mu} \partial_{\mu} \hat{h} \cdot \vec{\sigma}\right| \psi_{+}>}{2}\right| \psi_{-}>\right]
\end{aligned}
$$

Eq. (23) implies that

$$
<\psi_{-} \mid \partial_{\nu} \psi_{+}>=\frac{<\psi_{-}\left|\partial_{\nu} \hat{h} \cdot \vec{\sigma}\right| \psi_{+}>}{2} .
$$

As the result we have

$$
\begin{aligned}
& <\partial_{\nu} \psi_{+}\left|\psi_{-}><\psi_{-}\right| \partial_{\lambda} \psi_{+}>-[\nu \leftrightarrow \lambda] \\
& =\frac{1}{4}\left[<\psi_{+}\left|\partial_{\nu} \hat{h} \cdot \vec{\sigma}\right| \psi_{-}><\psi_{-}\left|\partial_{\lambda} \hat{h} \cdot \vec{\sigma}\right| \psi_{+}>-[\nu \leftrightarrow \lambda]\right. \\
& =\frac{1}{4} \sum_{n= \pm}\left[<\psi_{+}\left|\partial_{\nu} \hat{h} \cdot \vec{\sigma}\right| \psi_{n}><\psi_{n}\left|\partial_{\lambda} \hat{h} \cdot \vec{\sigma}\right| \psi_{+}>-[\nu \leftrightarrow \lambda]\right. \\
& =\frac{1}{4}\left[<\psi_{+}\left|\left[\partial_{\nu} \hat{h} \cdot \vec{\sigma}, \partial_{\lambda} \hat{h} \cdot \vec{\sigma}\right]\right| \psi_{+}>\right] \\
& =\frac{i}{2} \epsilon_{a b c}\left(\partial_{\nu} \hat{h}_{a}\right)\left(\partial_{\lambda} \hat{h}_{b}\right)\left[<\psi_{+}\left|\sigma_{c}\right| \psi_{+}>\right] \\
& =\frac{i}{2} \epsilon_{a b c}\left(\partial_{\nu} \hat{h}_{a}\right)\left(\partial_{\lambda} \hat{h}_{b}\right) \hat{h}_{c}=\frac{i}{2} \hat{h} \cdot\left(\partial_{\nu} \hat{h} \times \partial_{\lambda} \hat{h}\right) .
\end{aligned}
$$

In going from the second to the third line of Eq. (25) we have used the fact that $<\psi_{+}\left|\partial_{\nu} \hat{h} \cdot \vec{\sigma}\right| \psi_{+}><\psi_{+} \mid \partial_{\lambda} \hat{h}$. $\vec{\sigma} \mid \psi_{+}>-[\nu \leftrightarrow \lambda]=0$. Substituting Eq. (25) into Eq. (21) we obtain

$$
\begin{aligned}
B^{\mu} & =\epsilon^{\mu \nu \lambda} \operatorname{Im}\left[<\partial_{\nu} \psi_{ \pm} \mid \partial_{\lambda} \psi_{ \pm}>\right]=\frac{1}{4} \epsilon^{\mu \nu \lambda} \hat{h} \cdot \partial_{\nu} \hat{h} \times \partial_{\lambda} \hat{h} \\
& =2 \pi J^{\mu}
\end{aligned}
$$

After establishing Eq. (18) it is simple to prove (16) and (17). For (16) the Chern number is given by

$$
\mathcal{C}=\frac{1}{4 \pi} \int_{\mathcal{S}} d \mathbf{a} \cdot \mathbf{B}_{b}=\frac{1}{2} \int_{\mathcal{S}} d \mathbf{a} \cdot \mathbf{J}=\mathcal{P} / 2 .
$$

As the result $\mathcal{P}=n$ implies $\mathcal{C}=n / 2$. Now let us prove (17). Eq. (18) implies that

$$
\nabla \times \mathbf{A}_{b}=2 \pi \frac{1}{2 \pi} \nabla \times \mathbf{A}_{h}
$$

As the result $\mathbf{A}_{b}$ and $\mathbf{A}_{h}$ differ by a pure gauge at most

$$
\mathbf{A}_{b}=\mathbf{A}_{h}+\nabla \phi
$$

Since Eq. (12) is gauge invariant when $\mathcal{M}=R^{3} \bigcup\{\infty\}$, we conclude

$$
\begin{aligned}
\mathcal{C S} & =\frac{1}{4 \pi} \int_{S^{3}} d^{3} r \mathbf{A}_{b} \cdot \nabla \times \mathbf{A}_{b}=\frac{1}{4 \pi} \int_{S^{3}} d^{3} r \mathbf{A}_{h} \cdot \nabla \times \mathbf{A}_{h} \\
& =\mathcal{H} .
\end{aligned}
$$

As the result $\mathcal{H}=n$ implies $\mathcal{C S}=n$. 
In physics one often encounters Berry's phase when a system posses's "fast" and "slow" dynamic degrees of freedom. When the fast degrees of freedom are "integrated out" it often produces, as part of the effective action of the slow variables, a term (the Berry's phase) that is non-zero even when the slow variables change adiabatically with time. Such term can fundamentally alter the behavior of the slow variables.

In the following we present an example where the fast degrees of freedom generate an effective action that is the Hopf invariant of the slow variables. The model is a field theory in two space and one (Euclidean) time dimensions. It consists of two fields: 1$)$ a fermion field $\psi_{\sigma}(\mathbf{r}, t)$, and $2)$ an unit vector field $\hat{n}(\mathbf{r}, t)$. The Lagrangian density is given as

$$
\begin{aligned}
\mathcal{L} & =\mathcal{L}_{\psi}+\mathcal{L}_{n}-g \hat{n} \cdot \bar{\psi}_{\alpha} \vec{\sigma}_{\alpha \beta} \psi_{\beta} \\
\mathcal{L}_{\psi} & =\bar{\psi}_{\alpha}\left(\partial_{t}-\mu\right) \psi_{\alpha}-\frac{1}{2 m} \bar{\psi}_{\alpha}\left(\nabla-i \mathbf{A}_{e x}\right)^{2} \psi_{\alpha} \\
\mathcal{L}_{n} & =i \Omega[\hat{n}]+\frac{K}{2}|\nabla \hat{n}|^{2} .
\end{aligned}
$$

In the above $m, g, c, \mu$ are parameters of the model, $\mathbf{A}_{e x}$ is the vector potential of an external magnetic field, i.e., $\partial_{x} A_{y}-\partial_{y} A_{x}=B$, and $\delta \Omega / \delta \hat{n}=\hat{n} \times \partial_{t} \hat{n}$. Physically $\mathcal{L}_{\psi}$ describes fermions moving in an external magnetic field, and $\mathcal{L}_{n}$ describes the dynamics of magnetic moments in a ferromagnet. The last term in the first equation is the Zeeman coupling between the fermions and the magnetic moments. By adjusting $\mu$ we can tune the density $(\bar{\rho})$ of the fermions so that

$$
\bar{\rho}=k \frac{B}{\phi_{0}},
$$

where $\phi_{0}=2 \pi$ is the Dirac flux quantum and $k$ is an integer. When Eq. (32) is satisfied, the ground state of the fermions forms a so-called "integer-quantum Hall liquid". [8] Let us further assume that $g$ is large so that locally the electron spins have to be in the direction of $\hat{n}$. Under that condition integrating out the electrons produces a term $\frac{k}{4 \pi} \int d^{2} x d t \mathbf{A}_{h} \cdot \nabla \times \mathbf{A}_{h}$, which is proportional to the Hopf invariant of the $\hat{n}(\mathbf{r}, t)$. This term has the effect of changing the spins and statistics of solitons (the skyrmions) in the $\hat{n}(\mathbf{r}, t)$ field. [6]

Acknowledgements: DHL is in debt to Geoffrey Lee for helping him to visualize the dual of the Pontryagin form of the non-trivial Hopf map. We thank Qiang-Hua Wang for his help in making Figure 1. DHL is supported by NSF grant DMR 99-71503.

[1] M. Berry, Proc. R. Soc. London, A 392, 45 (1984).

[2] P.A.M. Dirac, Proc. Roy. Soc. A 133, 60 (1931).

[3] C.N. Yang, Ann. of N.Y. Acad. of Sci. 294, 86 (1977).

[4] S.S. Chern, Ann. of Math. 47, 85, (1946).

[5] S.S. Chern and J. Simoms Ann. Math. 99, 48 (1974).

[6] F. Wilczek and A. Zee, Phys. Rev. Lett. 52, 2111 (1984).

[7] See, e.g., R. Shankar, J. de. Phys. 38, 1405, (1977).

[8] For a review see, e.g., "The Quantum Hall Effect", edited by R.E. Prange and S.M. Girvin, (Springer-Verlag, New York 1986.)

\section{Figure Caption}

FIG. 1. The dual of the Pontryagin form in the central region of a $\mathcal{H}=1$ map (Eq. (15)). The green and blue arrows are the $\mathrm{x}, \mathrm{y}$ and $\mathrm{z}$ components of $\mathbf{J}$ respectively. 
This figure "pont.jpeg" is available in "jpeg" format from: http://arxiv.org/ps/cond-mat/0104099v1 